\title{
A Novel Maneuver for Colonic Polypectomy at the 6-0'Clock Position
}

For effective colonic polypectomy, the polyp has to be positioned in the 5-7o'clock position in the field of view. This has traditionally been achieved by changing the patient's position or by twisting the shaft of the scope, or both [1]. However, rotating a heavily sedated patient is not easy, and twisting the scope exerts a force on the shaft, the position of which has to be maintained by continually applying torque.

We have devised a simpler maneuver in which the orientation of the scope tip is adjusted to achieve proper alignment with the colonic polyp for polypectomy. To apply this "lift-the-scope" maneuver, it is important for the colonoscope to be straightened, with a length of less than $75 \mathrm{~cm}$ of the shaft inside the colon, so that a sufficient length of scope is outside the patient. The external portion of the shaft is rested on the examination table in such a way as to form a U-shape between the anus and the endoscopist (Figure $\mathbf{1}$ a).

The assistant lightly holds one of the two arms of the "U" about $15 \mathrm{~cm}$ from its apex and lifts it gradually from the examination table (Figure $\mathbf{1} \mathbf{b}$ ). During this maneuver, the polyp is seen to rotate around the field of view on the monitor, either clockwise when the patient's arm of the " $U$ " is lifted (i.e., the part running toward the patient's anus) or counterclockwise when the endoscopist's arm of the " $U$ " is lifted (the portion running toward the endoscopist). When a polyp is seen in the 7-912-o'clock positions, the endoscopist's arm of the " $U$ " should therefore be lifted; when a polyp is seen in the 12-3-5o'clock positions, the patient's arm of the "U" should be lifted.

We have calculated that this maneuver achieves the desired rotation of the scope while requiring 30 times less force in comparison with the classic twist-andtorque maneuver. During a formal validation of the lift-the-scope maneuver, 55\% of the polyps initially viewed in the 7 -
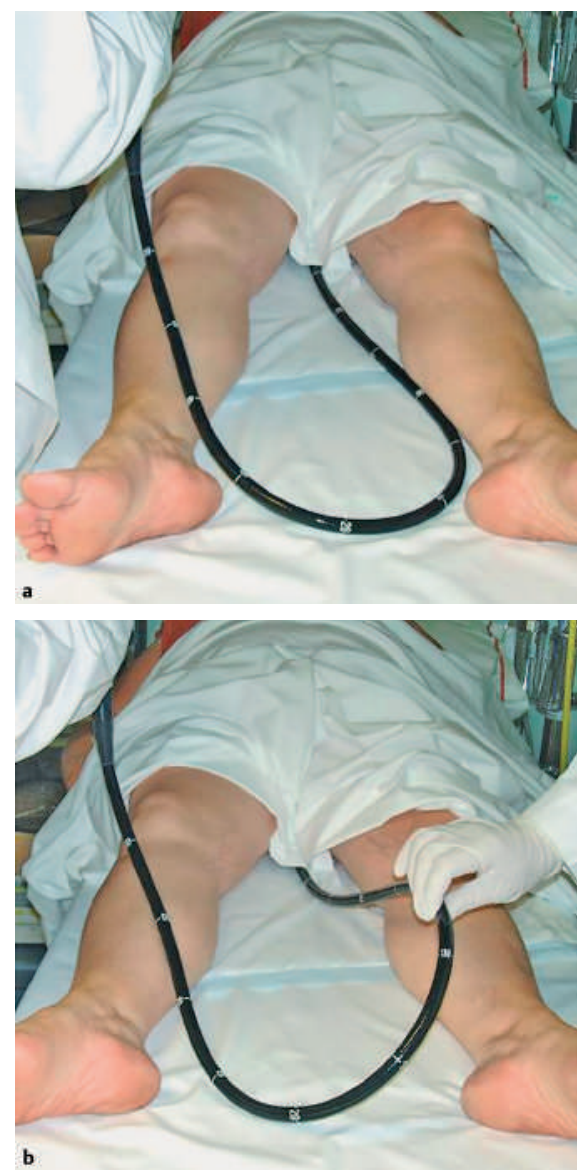

Figure 1 a With the patient in the supine position, the shaft of the colonoscope is rested on the examination table, forming a $\mathrm{U}$ shaped curve between the anus and the endoscopist. b When the right arm of the " $U$ "; is lifted from the examination table, the polyp is seen to move clockwise; when the left arm of the " $U$ " is raised, the polyp moves counterclockwise.

12-5-o'clock field of view even after the patient's position had been changed were easily placed in the 6-o'clock position.

\section{S. D. Ladas, C. Tzathas, D. Polymeros, T. Emmanuel, K. Triantafyllou} Hepatogastroenterology Unit, 2nd Dept. of Internal Medicine, Medical School of Athens University, Attikon University General Hospital, Athens, Greece.

\section{References}

${ }^{1}$ Cotton PB, Williams CB. Practical gastrointestinal endoscopy: the fundamentals, 5th ed.Oxford: Blackwell Science 2003

2 Waye JD. Colonoscopy intubation techniques without fluoroscopy. In: Hunt RH, Waye JD, (editors). Colonoscopy: techniques, clinical practice and color atlas.London: Chapman and Hall 1981: 147 - 178

\section{Corresponding Author}

\section{S. D. Ladas, M.D., Ph.D.}

Hepatogastroenterology Unit, 2nd Dept. of Internal Medicine Medical School of Athens University Attikon University General Hospital 23 Sisini Street

11528 Athens

Greece

Fax: $\quad+30-210-5326422$

E-mail: sdladas@hol.gr 